\title{
Reducing digger energy consumption by improving the walking mechanism
}

\author{
S. Lyaptsev ${ }^{1}$, N. Stepanova ${ }^{2} \&$ N. Suslov ${ }^{1}$ \\ ${ }^{1}$ Mining-mechanical Department, Ural State Mining University, Russia \\ ${ }^{2}$ Department of Finance, Ural Federal University, Russia
}

\begin{abstract}
To evaluate the energy to move the excavator body, we calculated the effort involved in the hydraulic cylinders of the walking mechanism. Unlike man, "pacing" the excavator is on a "drawing", in which the housing pulls the excavator walking mechanism in the direction of the reference points. As a result, the kinematic and dynamic study of the process of pacing is reduced to the analysis of speeds and loads for the simplest of mechanisms, known since ancient times as the slider-crank mechanism. Analysis of the motion of this mechanism can be carried out by conventional (calculated for one position) or by modern analytical methods. Modern methods of analytical studies of the mechanisms permit a computer calculation of the full cycle of movement of the excavator in a single step and to evaluate the stresses produced in the hydraulic cylinders. A variety of design solutions based on different positions of the connecting joints is recorded during the test set kinematics and, accordingly, an amount in the results is obtained. Power movement by one step excavator is theoretically estimated as the amount of work the forces acting on the mechanism during this step undertake. A design that corresponds to the minimum energy consumption should be considered optimal for this type of walking mechanism. The authors of this paper analyzed the performing over thirty different types of mechanisms of pacing. Recommendations for optimizing their designs are formulated for each of these, which not only reduces the power consumption excavator, but also reduces its price.
\end{abstract}

Keywords: excavator, walking mechanism, hydraulic cylinder, speed, acceleration, force, energy consumption. 


\section{Introduction}

A powerful digging machine, which is equipped with a special bucket for hard and soft rocks, is the excavator. Excavators are applied on open mining operations in pits, and also at extraction of fossil ores. Excavators can autonomously move by means of tracks or using a walking mechanism. A walking excavator is an excavator with a walking movement; it usually has dragline equipment. A dragline weight can reach many hundreds and even thousands of tons. The crawler mechanism is not applicable for heavy excavators due to the large deformations caterpillar tracks when the ground pressure is more than $2 \mathrm{~kg} / \mathrm{cm}^{2}$. The use of the walking hydraulic propulsion, unlike the crawler, reduces the load on the ground and improves the reliability of the excavator.

Currently, the most widely used are hydraulic excavators. In this type of dredging all the working parts are pivotally connected to the turntable and moved by hydraulic cylinders; each cylinder being driven by a high pressure pump. The greatest efforts must be made in the hydraulic cylinders of the walking mechanism, which permits movement of the body excavator. Such mechanisms are used for high mass excavating (over 800 tons).

A static excavator rests on the ground at the base of the base plate. If necessary to make a "step", weight is transferred to a shoe ("ski"). Each shoe is controlled by two pairs of hydraulic cylinders. Each of the hydraulic cylinders is setting in motion a high-pressure pump; oil pressure reaches $200 \mathrm{~atm}$. [1].

With a motion algorithm during walking: at the beginning the excavator rises above the ground; then it shifts some distance and sits back down on the ground by the base plate. The support shoes, in turn, are raised above the ground and are carried forwards. Then the cycle repeats. The actual speed of the excavator can be $60-80$ meters per hour; the maximum nameplate speed is up to $200 \mathrm{~m} / \mathrm{h}$.

The main functions of the walking equipment are:

- perception of machine weight and transferring the load to the ground;

- technological and transport movement of the machine;

- change of movement direction.

When moving the machine, the stepping walking equipment perceives a greater load of the strength of gravity, its point of application changes its position relative to the supports. Uneven distribution of gravity between the individual elements of the machine and the need to raise the machine to a considerable height greatly complicate the designing of walking mechanisms.

\section{Types of walking excavators}

Let us note the existing models used in the practice of mining on the example of two countries that are leaders in the designing of mining machine (Russia and America).

Four-support is the most powerful excavator walking mechanism [3] with complete separation of the base from the land (a scheme of the four-supporting stepping mechanisms is shown in fig. 1). The first time in Russia it was applied on a giant "ESH 100.100" weighing 11,000 tons, released in 1969. Now this 
excavator has been decommissioned and the current record belongs to "ESH 65.100", which works in the Tulun field. The biggest four-supporting excavators in the world are Big Maskie, of American construction. However, when it was designed, there was a defect as the heavyweight lifting body could not stand on the base and was deformed; the champion had to be dismantled.

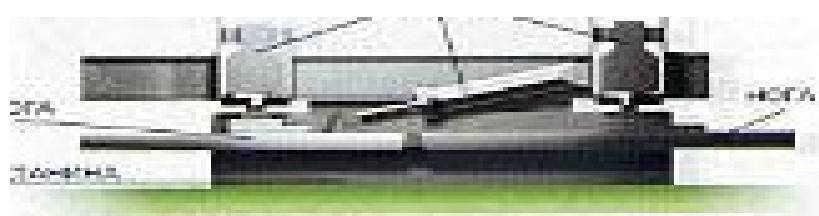

a) starting position

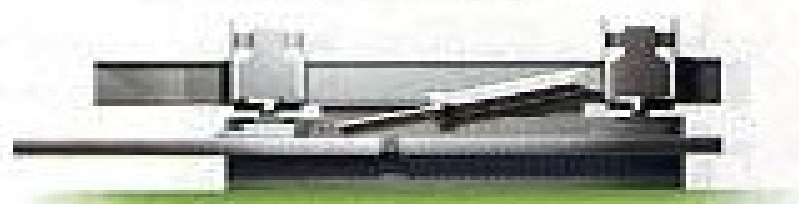

b) movement of the legs forward

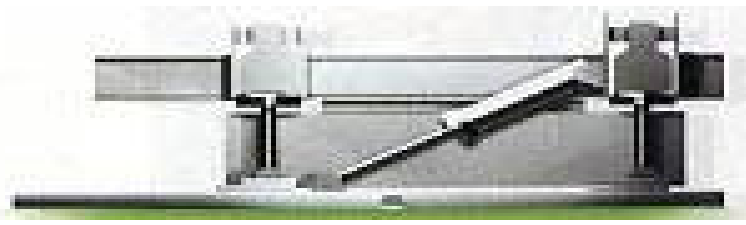

c) the rise of body

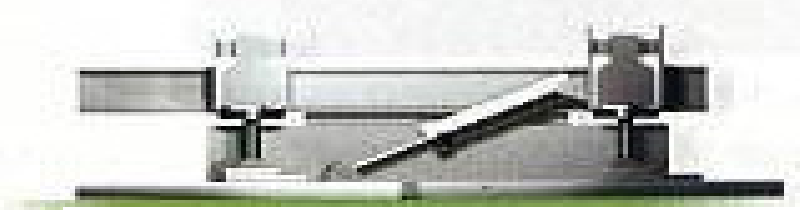

d) pulling up body

Figure 1: Scheme of movement of the four supporting mechanisms.

We note that Americans use mechanical eccentric drive stepping rather than hydraulic. The advantages of eccentric excavators are that they have more speed; up to $200 \mathrm{~m} / \mathrm{h}$ (instead of $80 \mathrm{~m} / \mathrm{h}$ in the hydraulic). The downside is that the tracks for them have to be much better prepared, otherwise the excavator may stall. Hydraulic legs can rise to different heights, and therefore slipping of Russian draglines is excluded. 
Three-support walking mechanisms are usually used for excavators of 1500 $3500 \mathrm{~kg}$ weight [4].

When an excavator with three-supporting stepping mechanisms is moving, it relies on two shoes and the nearest edge of the boom support base. Movement occurs in the opposite direction of the arrows.

The three-supporting excavators' manufacturer is the Russian company "OMZ (Uralmash-Izhora)".

A huge amount of energy is spent on moving the machine at high power loads due to its large mass. To evaluate the energy consumption and to develop measures for reducing it, it is necessary to carry out theoretical calculations which determine the kinematic and dynamic characteristics of the machines [2]. Theoretical calculations will also help to improve the walking mechanism so that energy consumption is minimized. Thus, to reduce the power consumption of the excavator it is necessary to study the kinematics and dynamics of walking mechanism and to choose the most economical construction of mechanism.

Unlike a human using two legs, the "step" of an excavator with three legs is "sliding": when the excavator is moving, its body is dragging itself towards the fulcrum by a stepping mechanism. As a result, the kinematic and dynamic study of the three-support stepping mechanism is reduced to the analysis of speeds, accelerations and loads for the simplest of mechanisms, known since ancient times, namely, crank mechanism. Analysis of the motion of this mechanism can be carried out by conventional methods (calculated for one position) or by modern analytical methods.

\section{Energy analysis of the walking mechanism}

Modern methods of analytical studies of the mechanisms permit a computer calculation of the full cycle of movement of the excavator in a single step and to evaluate the stresses produced in the hydraulic cylinders. A variety of design solutions based on different positions of the connecting joints is recorded in a plurality of the kinematic schemes investigated and, accordingly, an amount of the results are obtained.

Fig. 2 is a schema of the walking mechanism [5], which is an example of calculation shown below.

The mechanism includes shoes 1 placed on both sides of the body 2. Each side has three power cylinders and spacer arms 4 and 5. The levers are interconnected by the plunger 6 and the hinge 7, the footings and the platform are connected by hinges 8 and 9 . Angle limiter 10 bottom lever is installed between a lower spacer lever 5 and the supporting shoe. Levers 11 are connected to the platform 2 with stops 13 and serve to stabilize the translational movement of the shoes supported by the regulator 12. This execution of the walking mechanism significantly simplifies its design; it improves the drive control and increases the reliability of the excavator. 


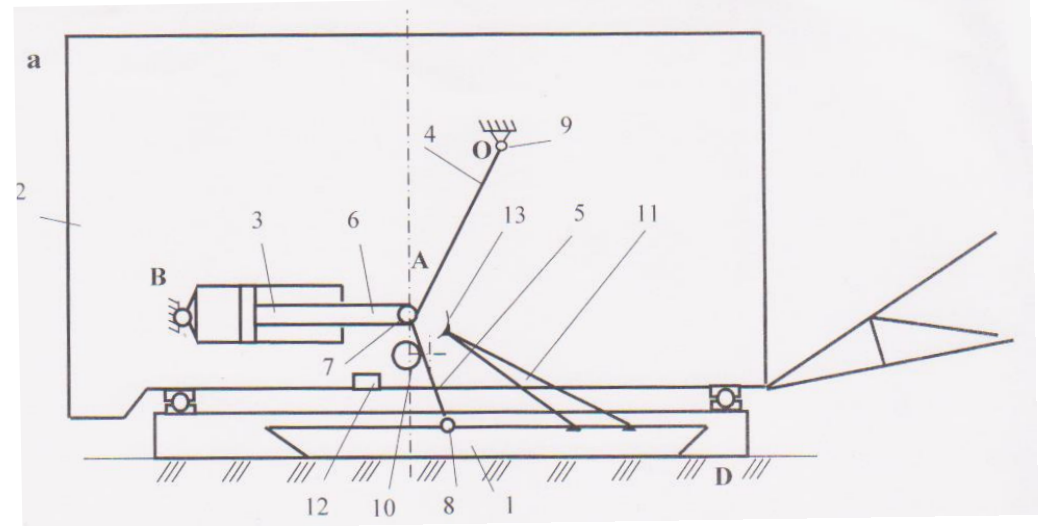

Figure 2: Scheme of walking mechanism.

To justify the parameters of the mechanism theoretical studies of this machine are undertaken $[6,7]$. This mechanism provides a horizontal movement for one pitch of about 2 meters. The fluid supplied to cylinder cavity during step action pushes the piston of the hydraulic cylinder at the same distance, by setting the displacement of point $\mathrm{A}$ in respect of the support.

The initial scheme is replaced by an equivalent kinematic scheme shown in fig. 3 .

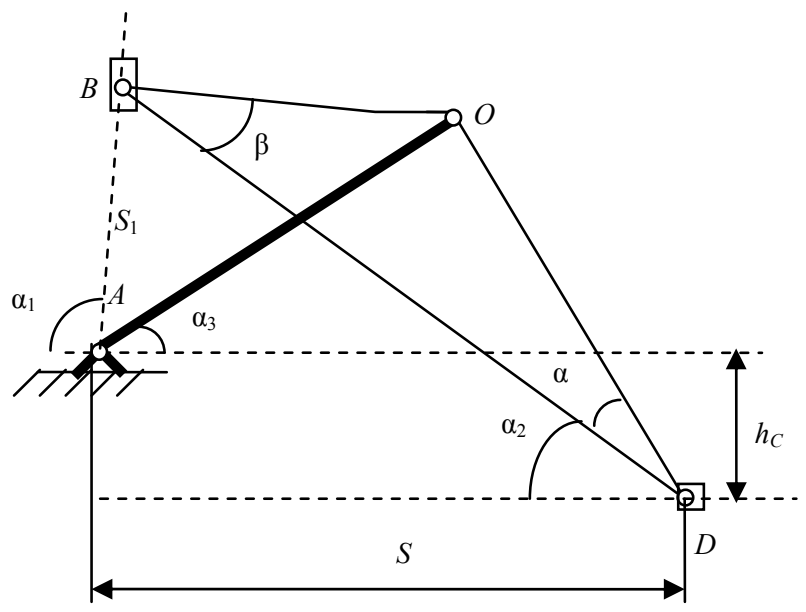

Figure 3: $\quad$ The equivalent kinematic scheme.

Kinematic equations of motion in accordance with this equivalent circuit are obtained by the vector method [2]: 


$$
\begin{gathered}
-S \cos \alpha_{1}+B O \cos \left(\beta-\alpha_{2}\right)-A O \cos \alpha_{3}=0, \\
S_{1} \sin \alpha_{1}+B O \sin \left(\beta-\alpha_{2}\right)-A O \sin \alpha_{3}=0, \\
-S_{1} \cos \alpha_{1}+B D \cos \alpha_{2}-S=0, \\
S_{1} \sin \alpha_{1}+B D \sin \alpha_{2}-h_{C}=0,
\end{gathered}
$$

where $S_{1}$ and $S$ - the move of the sliders $B$ и $D, \alpha, \beta, \alpha_{1}, \alpha_{2}, \alpha_{3}-$ the angles shown in fig. 3 .

Equations for analogs of velocities and accelerations are obtained by direct differentiation of (1) with respect to time:

$$
\begin{gathered}
-S_{1}^{\prime} \cos \alpha_{1}+S_{1} \sin \alpha_{1} \alpha_{1}{ }^{\prime}+B O \sin \left(\beta-\alpha_{2}\right) \alpha_{2}{ }^{\prime}+A O \sin \alpha_{3} \alpha_{2}{ }^{\prime}=0, \\
S_{1}{ }^{\prime} \sin \alpha_{1}+S_{1} \cos \alpha_{1} \alpha_{1}{ }^{\prime}-B O \cos \left(\beta-\alpha_{2}\right) \alpha_{2}{ }^{\prime}-A O \cos \alpha_{3} \alpha_{3}^{\prime}=0, \\
-S_{1}^{\prime} \cos \alpha_{1}-S_{1} \sin \alpha_{1} \alpha_{1}^{\prime}-B D \sin \alpha_{2} \alpha_{2}^{\prime}-S^{\prime}=0, \\
S_{1}^{\prime} \sin \alpha_{1}+S_{1} \cos \alpha_{1} \alpha_{1}^{\prime}-B D \cos \alpha_{2} \alpha_{2}^{\prime}=0 .
\end{gathered}
$$

(We do not give the equation for acceleration analogs in this article, as they are quite complex.)

The result is a system of algebraic equations, which allows us to determine both geometric and kinematic parameters. For example, when $O D=14 \mathrm{~m}$, $B D=18 \mathrm{~m}, B O=10.6 \mathrm{~m}, A O=11 \mathrm{~m}, h_{C}=4.2 \mathrm{~m}, S_{1}=A B=8 \mathrm{~m}$; can obtain: $\alpha_{1}=57^{0}, \alpha_{2}=38^{0}, \alpha_{3}=35^{0}$, Analogs of the angular velocity $\omega_{1}{ }^{*}=\omega_{1} / V_{1}=0.007 \mathrm{~m}^{-1}$, $\omega_{2}{ }^{*}=\omega_{2} / V_{1}=0.054 \mathrm{~m}^{-1}, \omega_{3}{ }^{*}=\omega_{3} / V_{1}=0.043 \mathrm{~m}^{-1}$, angular accelerations for $V_{1}=2 \mathrm{~m} / \mathrm{s}, \mathrm{a}_{1}=1 \mathrm{M} / \mathrm{c}^{2}$ equal to the following values $\varepsilon_{1}=0.075 \mathrm{~s}^{-2}, \varepsilon_{2}=0.57 \mathrm{~s}^{-2}$, $\varepsilon_{1}=0,14 \mathrm{~s}^{-2}$.

These results served as a control option for a computer program that solves the above system of nonlinear equations.

Element BOD was the research object under power analysis for a specified above kinematic scheme (see fig. 3). The equations of equilibrium for the system of active forces, reactions and inertial forces were drawn up in accordance with the principle of D'Elembert. Excavator mass is many times more its individual mobile units, so the force of gravity $m g$ is the only acting excavator force $(m-$ mass of excavator; $g$ - acceleration of gravity). Reactions at point $B$ consist of two components $X_{B}$ and $Y_{B}$, used in the point $D$ normal reaction $N$ and the friction force $F_{F R}=f n$, where $f$ - coefficient of friction. Inertial loads are the general vector and the general moment of forces of inertia $M^{I}=J_{C} \varepsilon_{1}$, where $J_{C}-$ moment of inertia of the excavator body. Equilibrium equations of the considered object have the form:

$$
\begin{aligned}
M^{I}+F_{O}^{\tau} A O+ & \left(F_{C O}^{\tau}-m g\right)\left(A O \cos \alpha_{3}+C O\right)+F_{F R} h_{C}+N\left[A O \cos \alpha_{3}+\right. \\
& \left.+O D \cos \left(\alpha+\alpha_{2}\right)\right]-F_{C O}^{n} \mathrm{AO} \sin \alpha_{3}=0
\end{aligned}
$$




$$
\begin{aligned}
&\left(N-m g+F_{C O}^{\tau}\right) \cos \alpha_{1}+\left(F_{F R}+F_{C O}^{n}\right) \sin \alpha_{1}-F_{O}^{\tau} \cos \left(\alpha_{1}+\alpha_{3}\right)+\left(F^{n}{ }_{O}-\right. \\
&-S) \sin \left(\alpha_{1}+\alpha_{2}\right)=0, \\
&-F_{F R} \cos \alpha_{1}+\left(F^{n}{ }_{O}-S\right) \cos \alpha_{3}+F_{O}^{\tau} \sin \alpha_{3}+F_{F R}+F_{C O}^{n}=0,
\end{aligned}
$$

where $F_{O}^{\tau}, F_{O}^{n}, F_{C O}^{\tau}, F_{C O}^{n}$ - the components of general vector inertia forces, which are expressed in terms of the corresponding components of acceleration.

Initial data for the complete calculation of the excavator step was selected numerical values corresponding draglines ESH 15.90A: $A O=5.5 \mathrm{~m}$, $B O=7.4 \mathrm{~m}, O D, O D=10 \mathrm{~m}, C \mathrm{O}=1 \mathrm{~m}, h_{C}=2 \mathrm{~m}$, the speed of the piston cylinder constant and equal to $V_{B}=0.5 \mathrm{~m} / \mathrm{s}$, excavator mass $m=1.6 \cdot 10^{6} \mathrm{~kg}$ coefficient of friction of the soil is assumed to be $f=0.5$. The calculation was performed for the condition of the extension of the piston cylinder so that at the initial time $A B_{0}=4 \mathrm{~m}$, and at the final moment $A B_{1}=6 \mathrm{~m}$. The results are shown

\begin{tabular}{|c|c|c|c|c|c|c|c|c|c|c|c|c|c|c|}
\hline$S_{1}$ & $\alpha_{1}$ & $\alpha_{2}$ & $\alpha_{3}$ & $S$ & $\omega_{1}$ & $\omega_{2}$ & $\omega_{3}$ & \multirow{2}{*}{\begin{tabular}{|c|}
$V_{D}$ \\
$\mathrm{~m} / \mathrm{s}$ \\
\end{tabular}} & $\varepsilon_{1}$ & $\varepsilon_{2}$ & $\varepsilon_{3}$ & \multirow{2}{*}{$\frac{a_{D}}{\mathrm{~m} / \mathrm{s}^{2}}$} & \multicolumn{2}{|c|}{\begin{tabular}{l|l}
$V$ & $F_{P}$
\end{tabular}} \\
\hline $\mathrm{M}$ & \multicolumn{3}{|c|}{ deg. } & $\mathrm{m}$ & \multicolumn{3}{|c|}{$\mathrm{s}^{-1}$} & & \multicolumn{3}{|c|}{$\frac{\mathrm{s}^{-2}}{2}$} & & \multicolumn{2}{|c|}{$\mathrm{MN}$} \\
\hline 4 & 1.01 & 9.94 & 78.65 & 7.62 & 1.43 & 0.13 & 0.79 & -0.52 & -5.45 & \begin{tabular}{|l|}
-0.64 \\
\end{tabular} & \begin{tabular}{|l|}
-0.96 \\
\end{tabular} & 0.09 & 0.36 & 0.38 \\
\hline 4.2 & 1.73 & 10.22 & 80.49 & 7.61 & 1.14 & 0.11 & 0.81 & -0.51 & -4.34 & $\mid-0.58$ & -0.51 & 0.06 & \begin{tabular}{|l|}
0.34 \\
\end{tabular} & 0.34 \\
\hline 4.4 & 2.27 & 10.44 & 82.36 & 7.40 & 0.87 & 0.09 & 0.82 & -0.50 & -3.45 & $\mid-0.56$ & \begin{tabular}{|l|}
-0.11 \\
\end{tabular} & 0.04 & 0.32 & 0.30 \\
\hline 4.6 & 2.65 & 10.63 & 84.27 & \begin{tabular}{|l|}
7.20 \\
\end{tabular} & 0.59 & 0.07 & 0.8 & -0.50 & -2.71 & \begin{tabular}{|l|}
-0.56 \\
\end{tabular} & 0.22 & 0.02 & \begin{tabular}{|l|}
0.29 \\
\end{tabular} & 0.27 \\
\hline 4.8 & 2.8 & 10.76 & 86.22 & 7.00 & 0.31 & 0.05 & 0.8 & -0.50 & -2.08 & -0.58 & \begin{tabular}{|l|}
0.49 \\
\end{tabular} & 0.01 & \begin{tabular}{|l|}
0.27 \\
\end{tabular} & 0.22 \\
\hline 5.0 & 2.95 & 10.85 & 88.22 & 6.79 & 0.02 & 0.02 & 0.8 & -0.50 & -1.53 & -0.63 & \begin{tabular}{|l|}
0.65 \\
\end{tabular} & 0.01 & 0.24 & 0.17 \\
\hline 5.2 & 2.89 & 10.87 & 90.28 & 6.59 & -0.29 & 0 & 0 & -0.51 & -1.01 & \begin{tabular}{|l|}
-0.69 \\
\end{tabular} & \begin{tabular}{|l|}
0.66 \\
\end{tabular} & 0.22 & 0.21 & 0.10 \\
\hline 5.4 & 2.69 & 10.83 & 92.41 & 6.39 & -0.62 & 0.03 & 0.94 & -0.51 & -0.49 & \begin{tabular}{|l|}
-0.76 \\
\end{tabular} & 0.46 & 0.03 & \begin{tabular}{|l|}
0.17 \\
\end{tabular} & 0.03 \\
\hline 5.6 & 2.36 & 10.72 & 94.62 & 6.19 & 0.97 & -0.06 & 0.98 & -0.51 & 0.12 & \begin{tabular}{|l|}
0.79 \\
\end{tabular} & $\mid-0.06$ & .06 & 0.12 & -0.05 \\
\hline 5.8 & 1.90 & 10.53 & 96.92 & 6.00 & -1.36 & -0.10 & 1.02 & -0.50 & 0.89 & \begin{tabular}{|l|}
-0.74 \\
\end{tabular} & $\mid-1.05$ & 0.12 & \begin{tabular}{|l|}
0.07 \\
\end{tabular} & -0.15 \\
\hline 6.0 & 1.29 & 10.25 & 99.31 & \begin{tabular}{|l|}
5.81 \\
\end{tabular} & -1.78 & -0.14 & 1.06 & $\begin{array}{l}-0.49 \\
\end{array}$ & 1.06 & \begin{tabular}{|l|}
-0.54 \\
\end{tabular} & \begin{tabular}{|l|}
-2.73 \\
\end{tabular} & 0.20 & \begin{tabular}{|l|}
0.01 \\
\end{tabular} & 0.28 \\
\hline
\end{tabular}
in table 1.

Table 1: Performance calculations of walking mechanism.

\section{The main results}

Analysis of the results shows:

1) The hydraulic cylinder retains almost horizontal position during the whole working stroke;

2) The excavator body does not change its original location;

3) The rocking body does not exceed 1-1.5 degrees;

4) The inclination angle of the lever $A O$ changes almost symmetrically with respect to the vertical and provides a step size excavator at about $2 \mathrm{~m}$;

5) The angular speed of the hydraulic cylinder, body of the excavator, lever $A O$ change slightly;

6) The angular accelerations are close to zero;

7) The sliding velocity at the point of the shoe matches the speed of movement of the piston cylinder, motion occurs almost evenly;

8) When the excavator body is moving, it retains its stable equilibrium; 
9) To ensure uniform motion of the piston cylinder and hence the excavator, the pressure in the hydraulic cylinder should change, and by the time step is finished, it should be to slow down the movement of the piston by liquid which is feeding in the opposite cavity;

10) The maximum force transmitted to the piston cylinder, is $0.38 \mathrm{MN}$, which, under normal pressure to $17.5 \mathrm{MPa}$ corresponds to the piston crosssectional area of $0.021 \mathrm{~m}^{2}$; its diameter should be chosen to be $0.16 \mathrm{~m}$;

11) In the case when the hydraulic cylinder contains a hydraulic cylinder with integrated additional dimensions of the main cylinder, its size will be even less. Metal consumption of the hydraulic cylinder in this case will be smaller; reducing the working pressure will increase the reliability of the pacing mechanism.

Power consumption for one step movement of the excavator is theoretically estimated as the sum of works of the forces acting on the mechanism for this step.

\section{Conclusion}

A constructive solution corresponding to the minimum energy consumption should be considered to be optimal for this type of walking mechanism.

The authors of this paper analyzed the performance of over thirty different types of mechanisms of pacing. Recommendations for optimizing their designs are formulated for each of these; it not only reduces the power consumption excavator, but also reduces its price.

\section{References}

[1] Poderni R. Yu. Walking draglines for open-cast mining, NII Informtyazhmash: Moscow, 70 p., 1980.

[2] Algin V.B. Dynamics, reliability and resource designing transmissions of mobile machines. Science and Technology: Minsk, 256 p., 1995.

[3] Alexander Grek. Walks with excavators: walking something. Popular Mechanics: Moscow, December 2002, p. 8.

[4] Gruzdev A. V., Boiko G. H. Draglines production corporation OMZ. Mining: Moscow, № 3, p. 16, 2003.

[5] Boyko G. H. Walking mechanism. Patent 1239229 (USSR): Moscow, publ. in IB № 23, 3 p., 1986.

[6] Suslov N. M., Lyaptsev S. A. Kinematic analysis and power walking mechanism of the excavator. Mining machines and automation, Moscow State Mining University: Moscow, № 7, pp. 9-11, 2004.

[7] Stepanova N. R., Suslov N. M. Kinematic analysis of the four-support walking mechanism of excavator. All-Russian Scientific and Technical Conference "Mathematical modeling of mechanical phenomena", Ural State Mining University: Ekaterinburg, pp. 88-95, 2013. 